SURVIE DE LA JEUNE ENTREPRISE :

UNE ETUDE DE CAS SUR LA BASE DES RELATIONS INTERPERSONNELLES

\begin{tabular}{|c|c|c|}
\hline Inès Gabarret & Gaël Bertrand & $\begin{array}{c}\text { Dominic Drillon } \\
\text { EDC Paris Business School }\end{array}$ \\
ines.gabarret@edcparis.edu & gSG Management School & $\begin{array}{c}\text { Goup de Co La } \\
\text { Rochelle }\end{array}$ \\
& & drillond@esc-larochelle.fr \\
\hline
\end{tabular}

\title{
RESUME
}

Cet article s'intéresse aux conditions de survie d'une jeune entreprise à partir de l'étude de la construction/destruction des liens sociaux unissant l'équipe entrepreneuriale. Beaucoup de travaux portent sur l'utilisation du réseau social pour le développement des entreprises, mais, quels sont les risques de mobiliser le réseau social pour trouver les bons associés pour une création d'entreprise ? La construction et le renforcement des liens entre les associés s'avèrent être des facteurs importants pour la survie et la réussite d'une jeune entreprise, notamment dans le cadre de la survie de l'équipe entrepreneuriale.

\section{MOTS CLES}

Survie, Jeune entreprise, Réseaux, Capital social 
SURVIE DE LA JEUNE ENTREPRISE :

UNE ETUDE DE CAS SUR LA BASE DES RELATIONS INTERPERSONNELLES

\section{RESUME}

Cet article s'intéresse aux conditions de survie d'une jeune entreprise à partir de l'étude de la construction/destruction des liens sociaux unissant l'équipe entrepreneuriale. Beaucoup de travaux portent sur l'utilisation du réseau social pour le développement des entreprises, mais, quels sont les risques de mobiliser le réseau social pour trouver les bons associés pour une création d'entreprise ? La construction et le renforcement des liens entre les associés s'avèrent être des facteurs importants pour la survie et la réussite d'une jeune entreprise, notamment dans le cadre de la survie de l'équipe entrepreneuriale.

\section{MOTS CLES}

Survie, Jeune entreprise, Réseaux, Capital social 


\section{INTRODUCTION}

La survie de la jeune entreprise est le critère minimum, la première étape, l'élément nécessaire dans l'accès au succès d'une entreprise (Tamàssy, 2006 ; Littunen, Storhammar et Nenonen, 1998). La recherche en entrepreneuriat s'oriente à considérer la survie comme une période qui va de 1 à 3 ans (Teurlai, 2004), temps qui correspond finalement à la durée présumée de la phase de démarrage (Sammut, 1998).

De la même façon que la survie nous conduit vers le succès de l'entreprise, la non survie (ou une cessation prématurée d'activité) nous met en relation avec le concept d'échec. Moreau (2007) nous indique qu'il n'est pas si aisé de différencier l'échec de la réussite. L'échec n'est pas seulement lié à des aspects financiers. Headd (2003) considère aussi des motifs propres aux propriétaires. Smida et Khelil (2010) proposent une typologie de huit catégories ou scenarii d'échec.

Pour assurer la réussite de la jeune entreprise, Baron (2000) explore le rôle des compétences sociales. La capacité à s'entendre avec les autres revêt une grande importance quand il s'agit de la survie d'une création d'entreprise.

Stam, Arzlanian et Elfring (2014) explicitent par ailleurs que l'activité entrepreneuriale : «is embedded in network relationships that direct resource flows to entrepreneurs who are somehow better connected... The social capital, or the resources that entrepreneurs may access through their personal networks allows entrepreneurs to identify opportunities, mobilize resources, and build legitimacy for their firms ».

Kamm et Schuman (1990) ou encore Eisenhardt et Schoonhoven (1990) ont établi un lien fort entre la réussite de l'entreprise et l'équipe fondatrice de la jeune entreprise sans pour autant expliquer la dynamique pouvant expliquer ce lien. Néanmoins, Bayad, Naffakhi et Schmitt (2007) montrent que les recherches entreprises sur ce type d'équipes se sont souvent limitées à l'exploitation des dynamiques d'équipe, tel que le processus optimal d'assemblage et de maintenance de l'équipe même ; sans pour autant tenter de lier ces éléments à la réussite ou à l'échec futur de la jeune entreprise.

Notre recherche se présente comme une étude de cas approfondie sur une création d'entreprise, menée à l'échec par la destruction des liens unissant l'équipe dirigeante. L'entreprise a été créée par trois associés qui se sont rencontrés en mobilisant leurs réseaux sociaux (leurs relations personnelles).

Dans un ouvrage, Danjou (2004) nous précise l'étymologie du mot entreprendre : «prendre ensemble ». C'est le cas sur lequel nous nous sommes penchés, celui d'une entreprise créée par trois associés. Ben-Hafaiedh (2006) utilise le terme «d'équipreneuriat» pour désigner ce phénomène d'entrepreneuriat en équipe ou collectif.

Par ailleurs, les entreprises peuvent être intégrées dans de multiples réseaux, focalisant souvent les recherches sur la place de l'entrepreneur au sein de ceux-ci amenant à considérer l'importance critique de ces liens pour le développement de l'entreprise (Stam, Arzlanian et Elfring, 2014).

Les réseaux sociaux permettent le développement du capital social, l'accès à l'information, la découverte d'opportunités, etc. Ils sont composés de liens faibles et de liens forts. Alors que les liens faibles permettent l'accès à une information plus élargie, les liens forts, basés sur des relations personnelles, améliorent la coopération entre les structures ou individus et la résolution de problèmes (Bollingtoft et Ulhoi, 2005).

Un autre aspect important repose sur la confiance entre chaque acteur, comprise comme la certitude que les autres feront bien les choses (Granovetter, 2005). 
Nous avons développé une étude qualitative, à visée exploratoire, basée sur des réunions avec l'équipe entrepreneuriale ainsi que sur des entretiens semi-structurés avec chaque entrepreneur à trois moments différents couvrant une période de deux ans. Nous avons analysé la création de liens au sein du groupe à partir de la mise en relation des associés, avant la création de l'entreprise, jusqu'à un an et demi après le début des activités.

Les résultats de la recherche montrent qu'une modification de l'équilibre dans lequel les liens sociaux se sont construits entre les associés peut être le déclencheur d'une destruction de ces liens et d'un échec dans la survie de l'entreprise ou d'un frein à son développement. A côté de la force des liens faibles (Granovetter, 1973) permettant l'accès à l'information nouvelle et aux opportunités, nous devons considérer aussi leur fragilité dans le cas des relations interpersonnelles. Il est important de renforcer les nouveaux liens, en développant la confiance, l'interaction et la connaissance mutuelle, car leur faiblesse peut conduire à une destruction des liens dans une équipe de travail, ceci mettant en péril la survie et la réussite de la jeune entreprise.

\section{LA SURVIE, LE SUCCES ET L'ECHEC DE L'ENTREPRISE}

La survie apparaît comme un indicateur nécessaire durant les premières phases d'existence de la jeune entreprise (phase de démarrage au sens de Sammut, 1998), pour appréhender dans les phases de développement suivant, le succès de la nouvelle entreprise. Tàmassy (2006) va dans le même sens et indique dans son étude que le critère minimum de succès qu'une entreprise peut escompter est la survie de celle-ci. Littunen, Storhammar et Nenonen (1998) sont plus tranchés sur la question en indiquant, dans un premier temps, que la survie est la première étape fondamentale dans l'accès au succès. Ils poursuivent en remarquant que du fait de la période de stabilisation de l'activité entrepreneuriale durant la phase d'émergence de la jeune entreprise, il est difficile de récolter suffisamment de données réellement fiables concernant la croissance et la profitabilité. Pour ces auteurs, la notion de survie apparaît donc comme l'élément nécessaire à l'obtention du succès. Il nous semble donc judicieux de considérer la survie comme l'élément initiant le processus conduisant la nouvelle entreprise à son succès futur, au même titre que Littunen, Storhammar et Nenonen (1998) ou encore Tamàssy (2006).

Considérer la notion de survie implique de prendre en compte un élément intrinsèque à cette notion, à savoir le temps. En effet, survivre implique une durée. Cette notion se distingue selon deux courants d'observations. Certains auteurs préféreront analyser le court ou moyen terme, alors que d'autres envisageront la notion de survie à long terme : la pérennité.

Brüderl, Preisendörfer et Ziegler (1992) analysent la survie durant les 5 premières années après la création de la jeune entreprise. Gartner, Starr et Bhat (1998), considèrent quant à eux que la survie à 4 ans est un indicateur clé dans le repérage du succès. Ciavarella, Buchholtz, Riordan, Gatewood et Stokes (2004) analysent la notion de survie au cours d'une étude longitudinale sur 8 ans. D'autres auteurs comme Singh (1997) axeront leur analyse sur l'observation de données relatives à 30 ans d'historique des entreprises étudiées. Le concept de pérennité apparaît comme le prolongement de la notion de survie, les deux analyses disposant de points d'observation différents, quant au stade de développement de l'entreprise étudiée. Les recherches sur ce sujet restent, cependant, peu nombreuses au regard du nombre de travaux existant sur la notion de survie.

Ainsi, la recherche en entrepreneuriat s'oriente plus généralement sur les études de la survie qui sont basées sur l'analyse à 1 et/ou 3 ans (Teurlai, 2004), temps qui correspond finalement à la durée présumée de la phase de démarrage (Sammut, 1998).

L'analyse de la survie suppose de prendre en compte la notion d'échec, en affinant ses contours par la distinction entre fermeture pour cause de faillite (échec) et fermeture pour cause de 
revente. Différents scenarii sont alors possibles, en considérant que l'entreprise qui ne survit pas n'est pas nécessairement en échec.

Headd (2003) insiste sur le fait qu'une entreprise est susceptible de cesser son activité (même si elle réussit financièrement) pour diverses raisons propres aux propriétaires ou stratégiques comme la décision d'être employé dans une entreprise qui rachèterait l'entreprise fermée.

Lussier (1995; Lussier et Pfeifer, 2000) axe quant à lui son analyse sur l'échec en faisant la distinction entre les firmes qui ont fermé de celles qui ont échoué. De plus, il considère le succès de manière incrémentale prenant la survie comme l'étape initiale d'un cheminement conduisant tout d'abord aux profits, puis enfin au succès.

Cochran (1981) distingue aussi 5 formes d'échecs, allant de la «banqueroute » à la «mort » de l'entreprise. Cette distinction lui permet de faire apparaitre un certain nombre de scenarii dans les cas où l'entreprise se retrouve fermée. Dans certains cas, elle ferme pour des causes financières, afin d'éviter les dettes ou tout du moins de les limiter. Dans d'autres cas, la fermeture peut se retrouver être une opportunité pour l'entreprise, cela revient à un rachat, une fusion ou encore une revente suivie d'une fermeture. Néanmoins, ces trois possibilités revêtent le même avantage, puisqu'elles reposent sur l'obtention d'une vente avec profit de l'entreprise. Enfin, l'auteur distingue un dernier scénario qui relève de la mort ou de la fermeture de l'entreprise.

La littérature traitant par ailleurs, de cette thématique, révèle également des modèles qui peuvent servir de base pour comprendre l'échec. L'examen des recherches antérieures révèle ainsi deux modélisations qui retiennent notre attention. Le modèle des «3E » de Paturel (1997, 2007) explique l'émergence d'une entreprise ou d'un projet viable par la convergence entre les aspirations du créateur (E1), les compétences et ressources intégrées à l'entreprise (E2) et les possibilités de l'environnement (E3).

Paturel (2007) va plus loin en développant un modèle d'analyse dit des «3F » et apporte un éclairage intéressant pour l'analyse de l'échec. Cette modélisation est conçue pour expliquer la performance des PME selon trois dimensions : l'efficacité (F1) consiste à rapprocher les objectifs réalisés des objectifs initialement fixés, l'efficience (F2) se rapporte à la manière dont les ressources et compétences sont utilisées pour réaliser les objectifs de l'entreprise, et l'effectivité (F3) qui concerne le niveau de satisfaction des parties prenantes de l'entreprise.

Smida et Khelil (2010) présentent une typologie de huit catégories ou scenarii d'échecs articulées autour de trois théories (théorie d'écologie des populations des organisations, théorie économique basée sur la primauté des ressources et la théorie psychologique de la brèche aspirations-réalisations). Autrement dit, ces auteurs prennent en compte trois aspects, le contexte entrepreneurial, les ressources et la motivation des entrepreneurs.

Des liens étroits existent entre les concepts de survie et de succès. En effet, survie et succès sont parfois confondus ou agglomérés dans leurs définitions. Certains auteurs tels que Baron (2000, 2003 avec Markman), Swierczek et Thanh (2003), ou encore Sternberg (2004), qui s'est basé sur les travaux de Baron (2000), ont axé leurs préoccupations sur l'existence de liens entre l'entrepreneur, ses capacités intellectuelles ou encore sa motivation et le succès futur de la jeune entreprise.

Baron en 2000, puis en 2003 avec Markman explorent le rôle des compétences sociales dans la réussite des entrepreneurs, ou en d'autres termes, "why the ability to get along with others really matters ». Cette citation se prête par ailleurs très bien à l'entreprise étudiée dans cette recherche. 
Le modèle qu'ils développent s'articule autour de trois variables fondamentales reflétant des compétences sociales singulières : (1) «The social perception », soit la capacité pour l'entrepreneur à comprendre son environnement, notamment les personnes qui y gravitent ; (2) "The social adaptability », soit la capacité pour l'entrepreneur de s'adapter dans les situations sociales diverses liées à l'exercice de sa fonction; et (3) «The expressiveness », soit la capacité pour l'entrepreneur d'être lui-même au cours des circonstances sociales qu'il rencontre.

Ce modèle d'analyse sur la capacité de l'entrepreneur à faire face à son environnement tente de montrer comment un entrepreneur peut orienter son comportement pour disposer d'un bon reflet vis-à-vis de son milieu, ce qui nous rapproche du concept de légitimité de l'entrepreneur par rapport à son environnement développé par Zimmerman et Zeitz (2002).

Ces différents développements ne sont pas sans rappeler un courant de recherche qui s'intéresse justement aux différentes catégories de capitaux que l'on peut qualifier de «sociaux » aussi bien pour l'entrepreneur que pour l'équipe entrepreneuriale, revenant à la capacité de ceux-ci à inter/ré/proagir avec leurs environnements respectifs, voire relativement à un environnement commun dans le cadre de la création d'entreprise.

Notre recherche nous mène vers l'articulation de deux notions fondamentales que sont la survie des jeunes entreprises et le capital social et humain de l'entrepreneur.

Une étude de Maes, Sels et Roodhooft (2003) a dévoilé l'importance du capital humain sur la profitabilité de la jeune entreprise. Ils ont montré que les caractéristiques du capital humain de l'entrepreneur avaient un effet direct sur la profitabilité, et aussi un effet indirect renforcé par des éléments de management.

Cette remarque se trouve ainsi dans la lignée des travaux de Enshassi et Liska (1999) ou encore de ceux de Sandivo, Grobler, Parfitt, Guvenis et Coyle (1992) qui spécifiaient que l'efficacité managériale du leader a un impact direct sur la productivité de l'équipe et stimule la motivation de la force de travail.

\section{LES RELATIONS INTERPERSONNELLES ET LE CONCEPT DE CAPITAL SOCIAL}

Il est de plus en plus admis par la communauté scientifique que l'activité entrepreneuriale intègre les relations sociales des réseaux des entrepreneurs qui leur permettent d'accéder plus facilement aux ressources dont ils ont besoin en étant d'une manière ou d'une autre mieux connectés de nos jours (Stam, Arzlanian et Elfring, 2014). La littérature indique clairement que le capital social, ou les ressources auxquelles les entrepreneurs pourraient avoir accès au travers de leur réseau personnel, permettent à l'entrepreneur d'identifier des opportunités (Bhagavatula, Elfring, Van Tilburg et Van De Bunt, 2010), de mobiliser des ressources et de construire la légitimité de leurs entreprises (Elfring et Hulsink, 2003 ; Zimmerman et Zeitz 2002).

Il est aussi connu que les réseaux sociaux ont une influence sur les résultats économiques. Un réseau est une structure sociale composée d'individus (ou organisations) appelés nœuds qui sont reliés par un ou plusieurs types d'interdépendances (professionnelle, amitié, parenté).

Les réseaux sociaux influencent le débit et la qualité de l'information, car les acteurs préfèrent faire confiance à des gens qu'ils connaissent. La confiance, définie par Granovetter (1985, 2005) comme la certitude que les autres feront bien les choses se développe à l'intérieur des réseaux. Les individus ont besoin de confiance. Granovetter (1985) ou encore Coleman (1988) vont même plus loin en considérant la notion de confiance comme une colle «that holds closely knit organizations together ». 
Coleman (1990) distingue trois types d'intermédiaires dans la confiance : les conseillers, les garants, et les entrepreneurs. Dans le rôle d'entrepreneur, l'intermédiaire induit la confiance de plusieurs individus et combine ses ressources. Bien que la confiance puisse être une ressource utile, elle est souvent fragile et insaisissable. Dans les milieux organisationnels, la confiance présente des effets positifs à l'égard de la réduction des coûts de transaction au sein des organisations, et par rapport à la sociabilité spontanée parmi les membres de l'organisation.

Dans l'étude des réseaux sociaux, les individus ne sont pas considérés comme étant isolés ou indépendants, et leurs choix ne sont pas purement rationnels sinon influencés par leurs liens sociaux (Laville, Levesque et This-Saint Jean, 2000). Un postulat principal de la théorie des réseaux sociaux repose sur l'existence de liens faibles et forts reliant les individus entre eux. La force d'un lien est une combinaison (probablement linéaire) de la quantité de temps, de l'intensité émotionnelle, de l'intimité (ou confiance mutuelle) et des services réciproques qui caractérisent ce lien. Plus les individus possèdent des liens forts, plus ils se ressemblent, plus ils partagent de l'information et de la connaissance. En complément, ce sont les liens faibles, les instruments indispensables pour saisir des opportunités.

Les différents types de liens vont donner accès à différents types de ressources (Granovetter, 1973, Stam, Arzlanian et Elfring, 2014). Les liens forts empêchent les actions opportunistes (bonding), en même temps qu'ils peuvent produire une fragmentation communautaire. Les liens faibles permettent l'accès à de nouvelles ressources, qui ne sont pas présentes dans leur réseau proche, et ainsi facilitent la saisie d'opportunités (bridging).

Alors que les liens faibles permettent l'accès à une information plus élargie, les liens forts basés sur des relations personnelles améliorent la coopération entre les structures ou individus et la résolution de problèmes (Bollingtoft et Ulhoi, 2005).

Les liens forts reposent sur le réseau de proximité (relation régulière, de confiance, parlant le même langage). Ces réseaux envoient des signaux forts. Les liens faibles permettent d'acquérir des informations nouvelles (réseaux à signaux faibles). Les signaux faibles remettent en cause le connu (Julien, 2000), permettent d'accélérer la vitesse d'acquisition de l'information et de réduire son coût (Cable et Shane, 2002).

L'analyse des réseaux sociaux a permis le développement de la théorie du capital social. Le capital social est l'ensemble des ressources qui sont liées à l'appartenance à un groupe ou à un réseau durable (Bourdieu, 1986). L'auteur le définit comme " made up of social obligations (connections), which is convertible, in certain conditions, into economic capital and may be institutionalized in the form of a title of nobility » (Bourdieu 1986, p. 243). Dans le même ordre d'idées, Burt (1992) considère qu'il est composé par les « friends, colleagues, and more general contacts through whom you receive opportunities to use your financial and human capital » (Burt 1992, p. 9).

James Coleman (1990) définit le capital social comme tout ce qui facilite l'action individuelle ou collective dans la société. L'auteur décrit plusieurs dimensions du capital social: (1) le relationnel : les relations de confiance et d'obligation sociale entre individus ; (2) la structure du réseau : le réseau de connaissances ; et (3) les normes : les normes et relations d'autorité entre individus. Dans la conception de Coleman, le capital social pourrait être bénéfique pour certaines personnes, alors que le même capital social peut être négatif pour d'autres. Le capital social peut être créé maintenu ou détruit (Coleman, 1990). Un facteur important qui affecte la création et la destruction du capital social est la stabilité de la structure sociale.

Presque toute forme de capital social dépend de la stabilité. Les perturbations de l'organisation sociale ou des relations sociales et la mobilité des individus peuvent être très destructrices pour le capital social. 
Pour Walker, Kogut et Shan (1997), le capital social peut être vu aussi bien comme une contrainte que comme une ressource. Le capital social contraint les normes de comportement entre les individus. Pour les auteurs, la coopération dans une relation ne peut se mettre en place sans une certaine obligation entre les différentes parties. Le cadre du capital social en contraignant la relation, assure son succès. Larson (1992) avait déjà mis en avant l'importance de la fonction de contrôle social des réseaux.

La théorie du capital social, dans ses 3 dimensions, prend en compte les aspects relationnels sous-jacents aux échanges entre les individus. Elle place le relationnel d'un individu comme un capital utile et utilisable, lequel s'ajoute à d'autres formes de capital comme le capital humain ou le capital économique. La théorie du capital social permet de considérer le réseau d'une personne ou d'une entreprise comme une ressource.

\section{METHODE DE RECHERCHE}

Cette étude, à vocation exploratoire et interprétative, est fondée sur une approche qualitative basée sur une étude de cas longitudinale. Cette méthode de recherche se base sur les travaux de Yin (1990) qui définissait l'étude de cas comme une enquête empirique qui étudie un phénomène contemporain dans son contexte réel, quand les frontières entre le phénomène et le contexte ne sont pas clairement évidentes, et dans lesquelles des sources multiples d'évidence sont employées. Yin (1990) souligne, aussi, que tout phénomène observé à travers une étude de cas unique peut avoir une portée générale.

La recherche a été basée sur une analyse approfondie de données collectées à trois moments différents sur une période de deux ans d'étude. Une première réunion de groupe a été organisée avec les futurs associés quelques mois avant la création de l'entreprise. Un deuxième contact, cette fois au travers d'entretiens individuels semi-structurés (Huberman et Miles, 1991) a été mené auprès des 3 associés, quelques mois après la création. Auparavant, nous avons préparé un guide avec les sujets principaux et sous-thèmes que nous voulions aborder lors de l'entrevue afin de comprendre les perceptions des personnes interrogées. Plus tard, à un an et demi du lancement de l'entreprise, un troisième contact a été réalisé, aussi dans la base d'entretiens individuels, afin de collecter les impressions de la première année de fonctionnement. Lors de ce dernier tour d'entretiens, la consigne était de raconter comment s'était passée la première année de vie de l'entreprise.

Les discours ont été enregistrés, retranscrits et évalués par une analyse de contenu (Bardin, 1977) qui est centrée sur une analyse thématique du discours (Evrard, Pras et Roux, 2003). L'approche interprétative, qui consiste à interpréter la parole en essayant de trouver un sens à percevoir ce qui est important pour la personne interrogée, semble particulièrement appropriée dans ce contexte.

Dans cette étude de cas, nous avons cherché à acquérir la perception la plus complète possible de l'objet de cette recherche à savoir la création d'entreprise en mobilisant le réseau social et les risques de la construction/destruction de liens entre les associés sur la survie d'une jeune entreprise ainsi que la relation de confiance entre chaque associé.

L'entreprise étudiée a été créée en 2010 pour commercialiser et installer des maisons en bois ou à ossature en bois, dans une optique de maison écologique. Trois associés se sont retrouvés liés dans cette création d'entreprise : Jean, Max et Paul. Le tableau 1 décrit les caractéristiques de chaque associé. 
Tableau 1 : L'équipe entrepreneuriale

\begin{tabular}{|c|c|c|c|}
\hline & JEAN & MAX & PAUL \\
\hline Education & $\mathrm{Bac}+8$ & $\mathrm{Bac}+5$ & $\mathrm{Bac}$ \\
\hline Nationalité & Français & Russe & Espagnol \\
\hline $\begin{array}{c}\text { Expérience } \\
\text { professionnelle }\end{array}$ & $\begin{array}{l}\text { Professeur et différentes } \\
\text { expériences professionnelles }\end{array}$ & $\begin{array}{l}\text { Commercial et Marketing } \\
\text { différentes sociétés }\end{array}$ & $\begin{array}{l}\text { Entrepreneur } \\
\text { maçonnerie. }\end{array}$ \\
\hline $\begin{array}{l}\text { Expérience dans le } \\
\text { secteur de la } \\
\text { création }\end{array}$ & Aucune & Très faible & Plus de 30 ans \\
\hline $\begin{array}{l}\text { Motivations et } \\
\text { objectifs pour ce } \\
\text { projet }\end{array}$ & $\begin{array}{l}\text { Possible reconversion suite à } \\
\text { une insatisfaction dans son } \\
\text { emploi, préparer sa fin de } \\
\text { carrière dans une entreprise qui } \\
\text { aurait du sens, en accord avec } \\
\text { ses valeurs }\end{array}$ & $\begin{array}{l}\text { Trouver un moyen de } \\
\text { subsistance suite à un } \\
\text { licenciement et ne } \\
\text { voulant pas déménager } \\
\text { pour trouver un autre } \\
\text { emploi }\end{array}$ & $\begin{array}{l}\text { Faire reprendre son } \\
\text { entreprise et préparer } \\
\text { la transmission, afin de } \\
\text { partir à la retraite }\end{array}$ \\
\hline$\%$ Parts sociales & 40 & 51 & 9 \\
\hline Statut & Associé & $\begin{array}{l}\text { Associé et Gérant de } \\
\text { l'entreprise }\end{array}$ & $\begin{array}{l}\text { Associé et salarié de } \\
\text { l'entreprise }\end{array}$ \\
\hline Rôle(s) initiaux & $\begin{array}{l}\text { Prospection de marché, relation } \\
\text { nouveaux clients, } \\
\text { communication et recherche sur } \\
\text { de nouveaux processus de } \\
\text { construction et de matériaux }\end{array}$ & $\begin{array}{l}\text { Administration, } \\
\text { marketing et achats } \\
\text { internationaux }\end{array}$ & $\begin{array}{l}\text { Maçonnerie et mise en } \\
\text { place des fondations } \\
\text { pour les installations } \\
\text { Maître d'œuvre }\end{array}$ \\
\hline Rôle(s) finaux & $\begin{array}{l}\text { Simple investisseur et soutien } \\
\text { ponctuel après son éloignement } \\
\text { pour raison professionnelle }\end{array}$ & $\begin{array}{l}\text { Démission } 1 \text { an après la } \\
\text { création, suite à } \\
\text { l'éloignement de Jean }\end{array}$ & $\begin{array}{l}\text { Gérant suite à la } \\
\text { démission de Max }\end{array}$ \\
\hline
\end{tabular}

\section{RESULTATS DE LA RECHERCHE}

L'analyse thématique des données sur la création et le développement de la première année d'activité de l'entreprise a permis l'identification de facteurs relationnels dans l'échec de la survie de l'entreprise. Ces derniers sont liés principalement à la forme dans laquelle les liens sociaux se sont construits entre les associés et à la perte de l'équilibre dans cette construction.

L'éloignement géographique d'un des associés, Jean, a créé un déséquilibre dans la composition des liens. Cet événement a constitué le déclencheur d'une destruction de liens et entrainé l'échec de la survie de l'entreprise. Ce changement dans le fonctionnement de l'équipe a eu pour conséquence une perte de confiance entre les partenaires. Des conflits et des problèmes internes surviennent, entraînant la démission d'un deuxième associé. À ce momentlà, il ne s'agit pas d'un éloignement géographique, mais d'une séparation effective. La composition de l'équipe a été détruite. La survie de l'entreprise est en péril.

L'évolution de la confiance dans l'équipe de direction de l'entreprise étudiée peut être comprise en deux phases. La première correspond à l'établissement de liens entre les partenaires sociaux, le développement de la confiance et la constitution de l'équipe entrepreneuriale. La deuxième phase commence avec des changements dans l'équipe. Cette phase entraine la destruction des liens de confiance entre les partenaires.

Les schémas ci-dessous résument les deux phases de cette étude. Le premier montre la manière dont les liens faibles et forts ont contribué à la constitution de l'équipe entrepreneuriale. Le deuxième représente les liens brisés qui ont eu lieu dans la deuxième période. 
Dans ces graphiques les lignes fines représentent les liens faibles, les lignes épaisses des liens forts, et les flèches rouges les nouvelles connexions. «E $\mathrm{E}$ est l'acteur externe qui a relié deux des entrepreneurs (le banquier qui a mis en contact Jean et Max). Jean, Max et Paul sont les trois associés.

Figure 1 : Création et destruction des liens
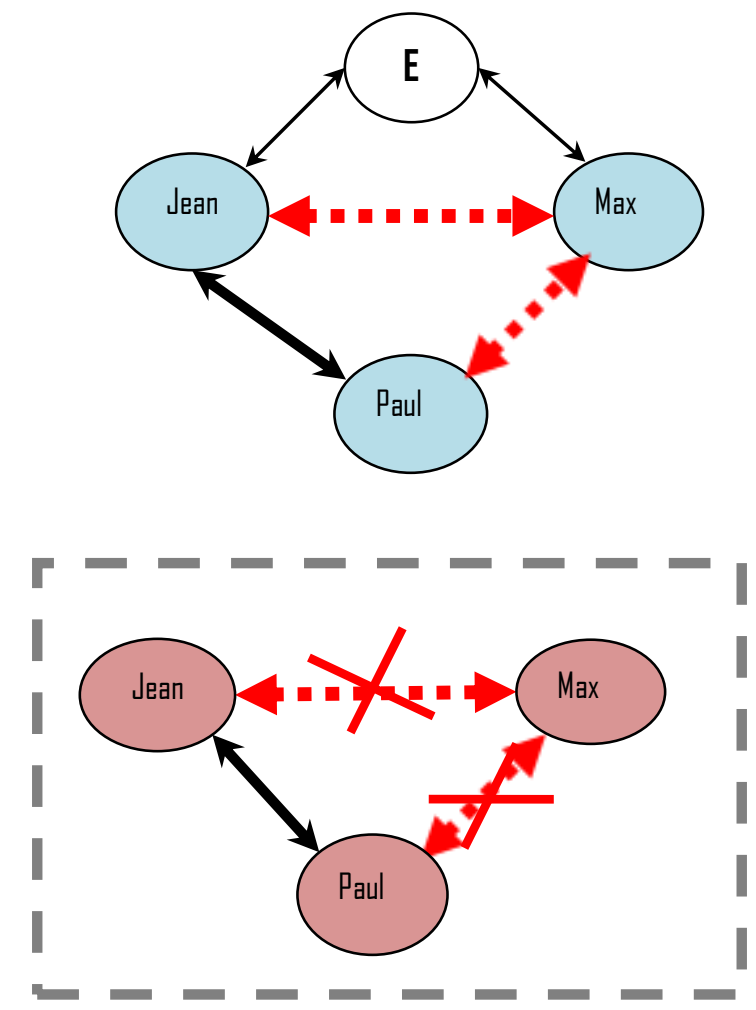

Comme on peut le voir, les relations brisées correspondent aux nouvelles connexions que l'on peut considérer comme de nouveaux liens faibles. Nous allons décrire le processus de construction et destruction des liens sociaux en 5 étapes, 3 correspondent à la phase de construction et 2 à celle de destruction.

\subsection{PREMIERE PHASE : La construction des liens sociaux}

\section{ETAPE 1 : Définition de l'idée : la rencontre de Jean et Max}

Dans cette première étape, le projet d'affaire et l'équipe entrepreneuriale commencent à prendre forme. Les premiers contacts dans la construction de l'équipe entrepreneuriale ont été faits avec l'intermédiation d'une troisième personne qui a mobilisé son réseau. Les deux premiers associés ont été présentés par un intermédiaire : leur banquier. Il s'agit d'une opportunité au travers des liens faibles, « bridging », tel que défini par Granovetter (1973) :

«Je m'intéressais au bois, à l'écologie et mon banquier me dit: comment se passe votre travail ? Et je lui réponds que j'aimerais faire autre chose, je voudrais construire des maisons en bois. Il me répond, c'est intéressant, est-ce que vous me permettez de donner vos coordonnées à un de mes clients qui s'intéresse à des projets d'énergie renouvelable ?»(Jean).

« J'ai rencontré Jean via notre banquier. Il voulait créer une entreprise écologique de construction de maisons en bois » (Max). 
Chabaud et Condor (2006) utilisent le terme de cristallisation du réseau social pour décrire comment deux ou plusieurs personnes s'organisent en équipe pour constituer une entreprise. Ces deux auteurs s'interrogent sur l'activation des liens forts et des liens faibles du porteur de projet.

Depuis le tout début, le choix des associés s'est fait en raison d'une confiance inspirée par l'appartenance à un même réseau de contacts (bancaire).

ETAPE 2 : Dépassement de la barrière d'entrée : l'incorporation de Paul

Cette étape est marquée par la recomposition du groupe entrepreneurial face à la présence d'une première barrière d'entrée au projet. Les futurs associés se voient dans l'impossibilité de faire bénéficier leurs premiers clients de l'assurance garantie décennale, pourtant obligatoire dans ce type d'entreprise. Ils décident donc d'incorporer un nouvel associé, en lui rachetant sa société :

«On a racheté l'entreprise de Paul pour obtenir cette garantie, si on avait pu avoir les assurances pour vendre des maisons en bois on n'aurait jamais racheté l'entreprise de Paul » (Max).

Le groupe entrepreneurial se complète ainsi en mobilisant, le capital social de Jean :

«J'avais parmi mes amis Paul, avec qui je faisais des randonnées, je lui explique ce projet, et il me dit : tu auras besoin d'un maçon pour construire les fondations de tes maisons, j'ai dit oui, il me rétorque, ne cherche plus, je suis là (...) on a étudié le montage du projet et finalement on a racheté l'entreprise de Paul tout en le conservant comme un troisième associé » (Jean).

« Finalement ce n'était plus une création mais une reprise de l'entreprise de Paul et la transformation de cette entreprise de maçonnerie en une entreprise de construction de maisons à ossature bois » (Max).

Cette intégration d'associés a été faite suivant la même logique de confiance qui a modelé le groupe entrepreneurial :

« Ma première impression de Paul, il est super sympa, on n'a pas de problème, mais après le travail c'est autre chose. Quand on s'est vu avec Jean et qu'on a signé les papiers, je l'avais vu 3 fois et on a signé, on a acheté l'entreprise sans passer du temps, sans voir les chiffres d'affaires, parce qu'il n'avait jamais déposé les chiffres d'affaires, c'était des estimations comme ça, à la louche et il aurait fallu travailler beaucoup plus pour avoir vraiment des informations » (Max).

ETAPE 3 : Début d'activités : une équipe entrepreneuriale qui fonctionne

Le début d'activités est marqué par une première vente et la distribution des tâches dans l'équipe entrepreneuriale. La confiance s'est installée entre les associés, ainsi que l'optimisme concernant le futur de l'entreprise :

« Notre travail premier, a été de constituer un réseau d'entrepreneurs, d'artisans, d'architectes,... maintenant nous avons notre premier client de maisons en bois, mais en ayant racheté l'entreprise de Paul, on a des travaux de maçonnerie qui nous permettront une diversification de nos activités et d'être plus viable économiquement » (Jean). 
« Cette entreprise, je la vois très bien, on a commencé très bien, nous avons beaucoup de clients, chaque fois qu'un client nous contacte il signe avec nous. Nous sommes dans une période de chance » (Paul).

«Pratiquement on a eu de la chance, on a eu tout de suite un client, un couple avec terrain qui a voulu construire une maison à ossature bois » (Max).

Les fonctions ont été distribuées parmi les associés : Jean est chargé de la prospection du marché, de trouver des nouveaux clients. Max s'occupe de l'administration de l'entreprise et de la gestion des achats à l'international, il a trouvé des fournisseurs en Europe. Paul s'occupe de gérer les travaux de maçonnerie pour la mise en place des fondations des maisons, ainsi que des travaux de finition. Max est nommé gérant de la société. Jean joue un rôle important dans l'équilibre et la stabilisation de l'équipe. Paul apporte ses compétences techniques et son expérience. Max tisse des liens avec des fournisseurs de Slovénie, le pays d'origine de ses beaux-parents.

\subsection{DEUXIEME PHASE : la destruction de liens sociaux}

ETAPE 1 : Eloignement de Jean, perte de l'équilibre et bouleversement

Peu de temps après la création de l'entreprise, un des associés (Jean) décide d'accepter une proposition d'emploi intéressante. Il déménage à plus de $600 \mathrm{~km}$, s'éloignant ainsi de la gestion de la société. De plus, ses nouvelles fonctions ne lui laissent pas de temps disponible pour s'occuper de l'entreprise :

«La reprise de cette entreprise n'avait pas amélioré mon insatisfaction professionnelle (...) j'ai continué à prospecter sur d'autres terrains et j'ai trouvé une proposition de salarié. Au départ je me disais c'est un peu dommage d'abandonner mes associés sur place (...) mais je vais créer très vite une antenne dans cette nouvelle ville où je serais partie prenante $\gg(J e a n)$.

L'éloignement de Jean est mal ressenti par les deux autres associés :

« S'il est parti c'est que Jean ne s'intéressait pas vraiment à l'entreprise et je n'aime pas ça. Il n'est jamais là, il ne demande pas comment vont les choses, j'aime que les gens soient actifs »(Paul).

«Jean est parti, pratiquement tout de suite après la vente de la première maison. On s'est retrouvé tout seul avec Paul à travailler dans l'entreprise » (Max).

Le départ de Jean a provoqué un changement dans l'équilibre établi entre les associés. Jean, qui était le lien entre Paul et Max, était aussi celui qui équilibrait les relations du groupe et qui légitimait les associés. Son départ représente une perte de cet équilibre et un nouveau système de forces se met en place. Max, le gérant, n'arrive pas à s'imposer face à Paul, qui retrouve tout son pouvoir informel sur l'entreprise une fois Jean parti :

«C'est vrai que j'ai un certain caractère, je ne supporte pas de patron au-dessus de moi, j'ai besoin de liberté, je sais bien ce que je fais » (Paul).

« Je ne maitrise pas les ouvriers, Paul a accepté de me laisser la gérance mais en réalité il fait ce qu'il veut (...) déjà, vis-à-vis du client je n'avais pas beaucoup de crédibilité, et en plus il prend des décisions sans me consulter, alors moi je sers à quoi ?» (Max). 
Ce départ a fragilisé l'équilibre entre les associés et donné lieu à un climat de méfiance. Le manque de confiance se trouve renforcé par des accusations mutuelles, et aussi par la culpabilisation de l'intermédiaire (Jean) dans le choix des associés :

«A l'époque l'entreprise je voulais la céder, et puis c'était Max, qui avait pris la gérance, moi je ne le connaissais pas puisque c'est Jean qui me l'avait présenté, donc je n'ai pas fait d'enquête sur lui, j'étais en confiance, mais aujourd'hui ça a commencé à dégénérer, il ne gère pas l'entreprise, il préfère partir en vacances et il ne s'occupe pas du chantier $\gg$ (Paul).

« Pourquoi Jean m'a sorti ce type là sans faire l'enquête, quand une entreprise prend un associé on fait un minimum d'enquête, pour savoir si la personne est sérieuse, quelles sont ses compétences, est-ce qu'il est apte à diriger une entreprise (...) J'ai accepté Max sous le conseil de Jean parce que moi je ne le connaissais pas » (Paul).

«J'aurais dû être plus méfiant, parce qu'il (Max) faisait de l'administration mais il faisait n'importe quoi » (Paul).

« J'ai sous-estimé les problèmes de société, Paul n'était pas prêt à laisser la gestion (...) J'avais un problème avec Paul parce que je disais il faut faire ça, ça et ça, et il me disait oui mais il ne le faisait pas. Et la réponse de Paul c'était : moi, j'ai 30 ans d'expérience et tu n'en as pas » (Max).

ETAPE 2 : Départ de Max, risque pour la survie de l'entreprise

Après un an de tensions, Max, le gérant, décide de quitter l'entreprise, faute d'appui de la part de Jean, et ne pouvant pas gérer Paul, l'associé minoritaire :

«Moi j'essaye de gérer au mieux, mais j'ai compris qu'avec Paul je n'y arrive pas. C'est lui la personne clé, sans lui je ne peux pas, mais avec lui je ne peux pas non plus. J'arrête parce que je n'y arrive plus » (Max).

«Comme ça j'ai dit j'arrête (...) parce que mes conditions étaient, je vais être gérant d'une entreprise, je dois faire confiance à mes partenaires. Jean est parti, il m'a planté là tout de suite et Paul je ne peux plus lui faire confiance » (Max).

Lors de la démission du gérant majoritaire (Max), le groupe entrepreneurial se trouve réduit à une seule personne (Paul), laquelle reprend la gérance :

« Il a démissionné (Max), il a laissé l'entreprise, il fallait que je la reprenne, je ne pouvais laisser l'entreprise à la dérive, c'est moi qui ai préparé l'assemblée extraordinaire, j'ai émis des réserves sur le bilan, et depuis il n'a jamais téléphoné une seule fois, pour prendre de nouvelles, pour savoir comment ça se passe, rien » (Paul).

Après l'éloignement de Jean, la démission de Max met l'entreprise en danger de mort. Paul, qui avait cherché à vendre son entreprise afin d'organiser son départ à la retraite se retrouve finalement dans la même situation qu'en début du projet. Il ne pourra pas assurer la continuité de l'entreprise et celle-ci risque de fermer. Max et Jean ont retrouvé des postes dans des entreprises et sont redevenus salariés.

Si l'on aborde ce cas selon l'angle des motivations individuelles, elles ne sont pas identiques lors de la constitution de l'entreprise. Après un an, la satisfaction que chaque associé peut en retirer est également différente. 
Tableau 2 : Motivation et satisfaction de l'équipe entrepreneuriale

\begin{tabular}{|c|l|l|}
\hline \multicolumn{1}{|c|}{$\begin{array}{l}\text { Motivation avant le démarrage de } \\
\text { l'entreprise }\end{array}$} & \multicolumn{1}{|c|}{ (In)Satisfaction après la création } \\
\hline JEAN & $\begin{array}{l}\text { Motivation non économique. Préparer sa } \\
\text { reconversion, s'engager dans cette } \\
\text { nouvelle activité en accord avec ses } \\
\text { valeurs }\end{array}$ & $\begin{array}{l}\text { Mitigée. Il n'a pas longtemps hésité à } \\
\text { saisir une opportunité professionnelle et } \\
\text { s'éloigner de ses associés. }\end{array}$ \\
\hline MAX & $\begin{array}{l}\text { Motivation par nécessité. Il doit } \\
\text { impérativement créer son emploi. Il a des } \\
\text { atouts pour développer cette activité. Ses } \\
\text { associés lui font confiance, il prend } \\
\text { naturellement la direction de l'entreprise }\end{array}$ & $\begin{array}{l}\text { Haute insatisfaction. Déçu de la réalité } \\
\text { du terrain. Il ressent très vite le décalage } \\
\text { entre ses aspirations et les difficultés du } \\
\text { métier quand ses associés lui refusent de } \\
\text { lui verser un salaire pour ne pas mettre } \\
\text { l'équilibre économique de l'entreprise en } \\
\text { danger. }\end{array}$ \\
\hline PAUL & $\begin{array}{l}\text { Motivation par opportunité. Il cherche à à } \\
\text { revendre son entreprise. C'est pour lui une } \\
\text { opportunité qu'il saisit. }\end{array}$ & $\begin{array}{l}\text { Faible insatisfaction. L'éloignement de } \\
\text { Jean et la démission de Max lui } \\
\text { redonnent le pouvoir, il reprend la } \\
\text { gérance. }\end{array}$ \\
\hline
\end{tabular}

\section{CONCLUSION}

Les résultats de cette recherche montrent l'importance d'un équilibre dans la composition des liens interpersonnels entre les associés afin de permettre la survie de l'entreprise. Les résultats vont dans le sens également de Sandivo, Grobler, Parfitt, Guvenis et Coyle (1992) lorsqu'ils précisaient pour le secteur de la construction l'importance du leader sur la productivité, la stimulation et la motivation de la force de travail. Dans notre cas, il est pertinent d'envisager que Jean occupait avant son éloignement une position de leader "soudant » les différents membres de l'équipe entrepreneuriale, les mettant dans la même direction. La dissolution des liens physiques est arrivée trop tôt, elle n'a pas permis à l'équipe entrepreneuriale de conserver son équilibre et son organisation fonctionnelle.

Cette recherche soulève la question de l'utilisation des réseaux pour créer des équipes d'entrepreneurs et souligne les risques de ce type de relations. Est-ce une bonne stratégie d'inviter de nouvelles personnes, inconnues, à travailler dans un projet, ou est-il préférable de former des équipes avec des personnes avec lesquelles il y existe une vraie connaissance préalable?

Ces résultats pourraient tout à fait aller dans le sens de Bayad, Naffakhi et Schmitt (2007) qui indiquent que les conflits au sein des équipes entrepreneuriales peuvent provenir de conflits de types affectifs entre les membres de l'équipe appartenant à des groupes sociaux différents. Ils montrent sur la base des travaux de Jehn (1997) que le conflit affectif ou relationnel provient d'un conflit personnel caractérisé par des frictions, des frustrations et des disputes entre les membres de l'équipe. Ils indiquent ainsi que ce genre de conflit peut arriver à nuire au bon fonctionnement de l'équipe engendrant une détérioration de l'esprit d'équipe. Ce propos nous amène à évoquer la question de la diversité dans le cadre de l'équipe entrepreneuriale. Selon ces auteurs, la diversité présente néanmoins un visage à double tranchant, engendrant d'une part une meilleure résolution des conflits, mais de l'autre créant parfois une barrière entre les membres de différentes disciplines, inhibant la coopération et générant une insatisfaction au 
sein de l'équipe. Cette notion pourrait, au sein de la dialogique hétérogénéité - homogénéité de l'équipe entrepreneuriale permettre d'affiner encore à l'avenir l'explication de la rupture au sein de l'équipe entrepreneuriale lorsque l'un des membres disparaît de l'échiquier entrepreneurial.

De leur côté, Bruyat et Julien (2000) affirment que le fait d'enlever un individu de l'équipe entrepreneuriale, supprimait ou changeait radicalement la dialogique individu/création de valeur nouvelle. Paturel (2005) évoque les équipes entrepreneuriales dans les termes suivants : «si l'un ou plusieurs de ses membres pourrai(en)t être sorti(s) de l'équipe sans dommage pour la réalisation du projet, ce ou ces membres ne sont pas entrepreneurs. » Pour ces auteurs l'équipe entrepreneuriale ne peut être modifiée sans entraîner le risque de destruction pour la jeune entreprise. Dans notre étude, les conséquences de l'éloignement de Jean confirment ce propos.

Les liens faibles pourraient permettre aux entrepreneurs de prospérer dans leurs projets, de découvrir de nouvelles opportunités, de constituer des équipes complémentaires, pour développer l'innovation, etc. Toutefois, les liens faibles sont aussi fragiles et peuvent se casser facilement si les conditions de confiance et de respect ne sont pas établies, et avoir des conséquences négatives sur l'avenir et la pérennité des projets.

Il est donc indispensable de lier l'utilisation des réseaux comme outils de développement avec d'autres activités pour renforcer les maillons faibles, afin de développer la compréhension mutuelle et d'aider les gens à mieux se connaître. Dans ces situations, le rôle de la personne qui permet le contact («bridge») est un facteur clé de succès pour la cohésion de l'équipe et la survie de l'entreprise.

\section{BIBLIOGRAPHIE}

BARDIN L. (1977), L'analyse de contenu, Presses Universitaires de France, Paris.

BARON R. A. (2000), "Psychological Perspectives on Entrepreneurship: Cognitive and Social Factors in Entrepreneurs' Success", Current Directions in Psychological Science, Vol. 9, $\mathrm{n}^{\circ} 1, \mathrm{p} .15-18$.

BARON R. A., MARKMAN G. D. (2003), "Beyond Social Capital: the Role of Entrepreneurs' Social Competence in their Financial Success", Journal of Business Venturing, Vol. 18, $\mathrm{n}^{\circ} 1, \mathrm{p} .41-60$.

BAYAD M., NAFFAKHI H., SCHMITT C. (2007), "L'équipe entrepreneuriale : rôle de la diversité dans le processus entrepreneurial", Actes du 5ème congrès International de l'académie de l'entrepreneuriat.

BEN-HAFAIEDH C. (2006), "Entrepreneuriat en équipe : positionnement dans le champ de l'entrepreneuriat collectif', Revue de l'entrepreneuriat, Vol. 5, p. 31-54.

BHAGAVATULA S., ELFRING T., VAN TILBURG A., VAN DE BUNT G.G. (2010), "How Social and Human Capital Influence Opportunity Recognition and Resource Mobilization in India's Handloom Industry", Journal of Business Venturing, Vol. 25, p. 245-260.

BOLLINGTOFT A., ULHOI J. P. (2005), "The Networked Business Incubator: Leveraging Entrepreneurial Agency?”, Journal of Business Venturing, Vol. 20, n 2, p. 265-290.

BOURDIEU P. (1986), "The Forms of Capital”, in J. Richardson (ed.), Handbook of Theory and Research for the Sociology of Education, New York, Greenwood, p. 241-258.

BRÜDERL J., PREISENDORFER P., ZIEGLER R. (1992), "Survival Chances of Newly Founded Business Organizations", American Sociological Review, 57, 2 p. 227.

BRUYAT C., JULIEN P. A. (2000), "Defining the Field of Research in Entrepreneurship", Journal of Business Venturing, Vol. 16, n 2, p. 165-180. 
BURT R.S. (1992), Structural Holes: The Social Structure of Competition, Cambridge, MA, Harvard University Press.

CABLE D., SHANE S. (2002), "Network Ties, Reputation, and the Financing of New Ventures", Management Science, March, Vol. 48, n 3, p. 364-381.

CHABAUD D., CONDOR R. (2006), "Le rôle des réseaux dans le processus de création d'entreprises : faut-il compléter la perspective ?", XVième conférence de l'AIMS Annecy.

CIAVARELlA M. A., BUCHHOLTZ A. K., RIORDAN C. M., GATEWOOD R. D., STOKES G. S. (2004), "The Big Five and Venture Survival: Is There a Linkage?", Journal of Business Venturing, Vol. 19, p. 465-483.

COCHRAN A. B. (1981), "Small Business Mortality Rates: A Review of the Literature", Journal of Small Business Management, (pre-1986), Vol. 19, $\mathrm{n}^{\circ}$ 4, p. 50.

COLEMAN J. (1988), "Social Capital in the Creation of Human Capital", American Journal of Sociology, Vol. 94, p. 95-120.

COLEMAN J. (1990), Foundations of Social Theory, Cambridge, MA, Harvard University Press.

DANJOU I. (2004), Entreprendre : la passion d'accomplir ensemble, L'Harmattan, Paris.

EISENHARDT K. M., SCHOONHOVEN C. B. (1990), "Organizational Growth: Linking Founding Team, Strategy, Environment, and Growth among U.S. Semiconductor Ventures", 1978-1988, Administrative Science Quarterly, Vol. 35, n 3, p. 504-29.

ELFRING T., HULSINK W. (2003), "Networks in Entrepreneurship: The Case of HighTechnology Firms", Small Business Economics, Vol. 21, p. 409-422.

ENSHASSI A., LISKA R. (1999), "Comparison between the Leadership Style of American and Palestinian Construction Managers", Joint Trienial Symposium, Customer Satisfaction; A Focus for Research \& Practice, Cape Town: 5-10 September 1999, Editors: Bowen P. \& Hindle R.

EVRARD Y., PRAS B., ROUX E. (2003), Market: Etudes et recherches en marketing, Dunod.

GARTNER W. B., STARR J. A., BHAT S. (1998), "Predicting New Venture Survival: An Analysis of " Anatomy of a Start-up"”, Journal of Business Venturing, Vol. 14.

GRANOVETTER M. (1973), "The Strength of Weak Ties", American Journal of Sociology, Vol. 78, n 6, p. 1360-1380.

GRANOVETTER M. (1985), "Economic Action and Social Structure: the Problem of Embeddedness", American Journal of Sociology, Vol. 91, n 3, p. 481-510.

GRANOVETTER M. (2005), "The Impact of Social Structure on Economic Outcomes", Journal of Economic Perspectives, Vol. 19, $\mathrm{n}^{\circ}$ 1, p. 33-50.

HEADD B. (2003), "Redefining Business Success: Distinguishing between Closure and Failure", Small Business Economics, vol. 21, n 1, p. 51.

HUBERMAN A., MILES M. (1991), Analyse des données qualitatives. Recueil de nouvelles methods, De Boeck Université.

JEHN K. A. (1997), "A Qualitative Analysis of Conflict Types and Dimensions in Organizational Groups", Administrative Science Quarterly, Vol. 42, p. 530-557.

JULIEN P. A. (2000), "Régions dynamiques et PME à forte croissance : Incertitude, information potentielle et réseaux à signaux faibles", en Histoire d'entreprendre : les réalités de l'entrepreneuriat, dirigé par T. Verstraete, EMS, p. 49-66.

KAMM J. B., SHUMAN J. C. (1990), "Entrepreneurial Teams in New Venture Creation", Entrepreneurship Theory and Practice, Vol. 14, p. 7-24.

LARSON A. (1992), "Network Dyads in Entrepreneurial Settings: A Study of the Governance of Exchange Relationships", Administrative Science Quarterly, Vol. 37, p.76-104. 
LAVILLE J. L., LEVESQUE B., THIS-SAINT JEAN I. (2000), "La dimension sociale de l'économie selon Granovetter", dans Le marché autrement : Essais de Mark Granovetter, collection Sociologie économique, p.9-32.

LITTUNEN H., STORHAMMAR E., NENONEN T. (1998), "The Survival of Firms over the Critical First 3 Years and the Local Environment", Entrepreneurship \& Regional Development, Vol. 10, $\mathrm{n}^{\circ} 3$, p. 189-202.

LUSSIER R. N. (1995), "A Nonfinancial Business Success versus Failure Prediction Model”, Journal of Small Business Management, Vol. 33, $\mathrm{n}^{\circ} 1, \mathrm{p} .8$.

LUSSIER R. N., PFEIFER S. (2000), "A Comparison of Business Success versus Failure Variables between U.S. and Central Eastern Europe Croatian Entrepreneurs", Entrepreneurship: Theory \& Practice, summer, p. 9.

MAES J., SELS L., ROODHOOFT F. (2003), "Modeling Small Business Profitability. An Empirical Test in the Construction Industry", Working Paper Steunpunt OOI: August 2003

MOREAU R. (2007), "La réussite entrepreneuriale : proposition pour un modèle explicatif general", Gestion 2000, Vol. 24, n 3, p. 45-62.

PATUREL R. (1997), Pratique du management stratégique, Presses Universitaires de Grenoble.

PATUREL R. (2005), "Pistes de réflexions en vue de l'élaboration d'une grille de positionnement des pratiques entrepreneuriales", 4e congrès de l'Académie de l'Entrepreneuriat, Paris, actes.

PATUREL R. (2007), Dynamiques entrepreneuriales et développement économique, L'Harmattan, Paris, Collection Mouvements Economiques et Sociaux.

SAMMUT S. (1998), Jeune Entreprise. La phase cruciale du démarrage, L'Harmattan, Paris.

SANDIVO V., GROBLER F., PARFITT K., GUVENIS M., COYLE M. (1992), "Critical Success Factors for Construction Projects", Journal of construction engineering and management, Vol. 118, $\mathrm{n}^{\circ}$ 1, p. 94-111.

SINGH K. (1997), "The Impact of Technological Complexity and Interfirm Cooperation on Business Survival", Academy of Management Journal, Vol. 40, n 2, p. 339-367.

SMIDA A., KHELIL N. (2010), "Repenser l'échec entrepreneurial des petites entreprises émergentes. Proposition d'une typologie s'appuyant sur une approche integrative", Revue Internationale P. M. E., Vol. 23, $\mathrm{n}^{\circ}$ 2, p. 65-105.

STAM W., ARZLANIAN S., ELFRING T. (2014), "Social Capital of Entrepreneurs and Small Firm Performance: A Meta-Analysis of Contextual and Methodological Moderators", Journal of Business Venturing, Vol. 29, p. 152-173.

STERNBERG R. J. (2004), "Successful Intelligence as a Basis for Entrepreneurship", Journal of Business Venturing, Vol. 19, $\mathrm{n}^{\circ}$ 2, p. 189-201.

SWIERCZEK F. W., THANH H. T. (2003), "Motivation, Entrepreneurship and the Performance of SMEs in Vietnam", Journal of Enterprising Culture, Vol. 11, n 1, p. 4768.

TAMÀSSY C. (2006), "Determinants of Regional Entrepreneurship Dynamics in Contemporary Germany: A Conceptual and Empirical Analysis", Regional Studies, Vol. $40, n^{\circ} 4$, p. 20.

TEURLAI J. C. (2004), "Comment modéliser les déterminants de la survie et de la croissance des jeunes entreprises ?", Cahier de recherche CRÉDOC, n¹97, février.

WALKER G., KOGUT B., SHANE W. (1997), "Social Capital, Structural Holes and the Formation of Industry Network", Organisation Science, Vol. 8, n² 2, p. 109-125.

YIN R. (1990), Case Study Research: Design and Methods, Thousand Oaks, CA, Sage Publishing.

ZIMMERMAN M., ZEITZ G. (2002), "Beyond Survival: Achieving New Venture Growth by Building Legitimacy", The Academy of Management Review, Vol. 27, n 3, p. 414. 